\title{
Semantic Alignment: Finding Semantically Consistent Ground-truth for Facial Landmark Detection
}

\author{
Zhiwei Liu ${ }^{1,2 *}$ Xiangyu Zhu ${ }^{1 *}$, Guosheng $\mathrm{Hu}^{4,5}$, Haiyun Guo ${ }^{1}$, Ming Tang ${ }^{1,3}$, Zhen Lei ${ }^{1}$, Neil M. Robertson ${ }^{5,4}$ and Jinqiao Wang ${ }^{1,3}$ \\ ${ }^{1}$ National Lab of Pattern Recognition, Institute of Automation, CAS, Beijing 100190, China ${ }^{2}$ University of Chinese \\ Academy of Sciences ${ }^{3}$ Visionfinity Inc., ObjectEye Inc., Universal AI Inc. ${ }^{4}$ AnyVision ${ }^{5}$ Queens University Belfast \\ ${ }^{1}$ \{zhiwei.liu, xiangyu.zhu, haiyun.guo, tangm, zlei, jqwang\}@nlpr.ia.ac.cn \\ ${ }^{4}$ huguosheng100@gmail.com ${ }^{5} \mathrm{~N}$. Robertson@qub.ac.uk
}

\begin{abstract}
Recently, deep learning based facial landmark detection has achieved great success. Despite this, we notice that the semantic ambiguity greatly degrades the detection performance. Specifically, the semantic ambiguity means that some landmarks (e.g. those evenly distributed along the face contour) do not have clear and accurate definition, causing inconsistent annotations by annotators. Accordingly, these inconsistent annotations, which are usually provided by public databases, commonly work as the groundtruth to supervise network training, leading to the degraded accuracy. To our knowledge, little research has investigated this problem. In this paper, we propose a novel probabilistic model which introduces a latent variable, i.e. the 'real' ground-truth which is semantically consistent, to optimize. This framework couples two parts (1) training landmark detection CNN and (2) searching the 'real' groundtruth. These two parts are alternatively optimized: the searched 'real' ground-truth supervises the CNN training; and the trained CNN assists the searching of 'real' groundtruth. In addition, to recover the unconfidently predicted landmarks due to occlusion and low quality, we propose a global heatmap correction unit (GHCU) to correct outliers by considering the global face shape as a constraint. Extensive experiments on both image-based ( $300 \mathrm{~W}$ and AFLW) and video-based $(300-V W)$ databases demonstrate that our method effectively improves the landmark detection accuracy and achieves the state of the art performance.
\end{abstract}

\section{Introduction}

Deep learning methods [23, 31, 34, 13, 7, 26] have achieved great success on landmark detection due to the strong modeling capacity. Despite this success, precise and credible landmark detection still has many challenges, one

\footnotetext{
* equal contribution.
}

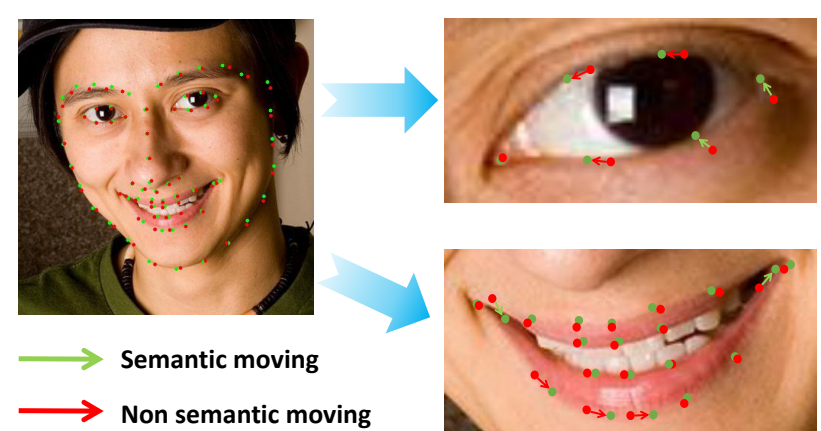

Figure 1. The landmark updates in training after the model is roughly converged. Due to 'semantic ambiguity', we can see that many optimization directions, which are random guided by random annotation noises along with the contour and 'non semantic'. The others move to the semantically accurate positions. Red and green dots denote the predicted and annotation landmarks, respectively.

of which is the degraded performance caused by 'semantic ambiguity'. This ambiguity results from the lack of clear definition on those weak semantic landmarks on the contours (e.g. those on face contour and nose bridge). In comparison, strong semantic landmarks on the corners (e.g. eye corner) suffer less from such ambiguity. The 'semantic ambiguity' can make human annotators confused about the positions of weak semantic points, and it is inevitable for annotators to introduce random noises during annotating. The inconsistent and imprecise annotations can mislead CNN training and cause degraded performance. Specifically, when the deep model roughly converges to the ground-truth provided by public databases, the network training is misguided by random annotation noises caused by 'semantic ambiguity', shown in Fig. 1. Clearly these noises can make the network training trapped into local minima, leading to degraded results.

In this paper, we propose a novel Semantic Alignment method which reduces the 'semantic ambiguity' intrinsi- 
cally. We assume that there exist 'real' ground-truths which are semantically consistent and more accurate than human annotations provided by databases. We model the 'real' ground-truth as a latent variable to optimize, and the optimized 'real' ground-truth then supervises the landmark detection network training. Accordingly, we propose a probabilistic model which can simultaneously search the 'real' ground-truth and train the landmark detection network in an end-to-end way. In this probabilistic model, the prior model is to constrain the latent variable to be close to the observations of the 'real' ground truth, one of which is the human annotation. The likelihood model is to reduce the Pearson Chi-square distance between the expected and the predicted distributions of 'real' ground-truth. The heatmap generated by the hourglass architecture [17] represents the confidence of each pixel and this confidence distribution is used to model the predicted distribution of likelihood. Apart from the proposed probabilistic framework, we further propose a global heatmap correction unit (GHCU) which maintains the global face shape constraint and recovers the unconfidently predicted landmarks caused by challenging factors such as occlusions and low resolution of images. We conduct experiments on 300W [21], AFLW [9] and 300VW [22, 24, 3] databases and achieve the state of the art performance.

\section{Related work}

In recent years, convolutional neural networks (CNN) achieves very impressive results on many computer vision tasks including face alignment. Sun et al [23] proposes to cascade several DCNN to predict the shape stage by stage. Zhang et al [30] proposes a single CNN and jointly optimizes facial landmark detection together with facial attribute recognition, further enhancing the speed and performance. The methods above use shallow CNN models to directly regress facial landmarks, which are difficult to cope the complex task with dense landmarks and large pose variations.

To further improve the performance, many popular semantic segmentation and human pose estimation frameworks are used for face alignment [29, 5, 2, 14]. For each landmark, they predict a heatmap which contains the probability of the corresponding landmark. Yang et al. [29] uses a two parts network, i.e., a supervised transformation to normalize faces and a stacked hourglass network [17] to get prediction heatmaps. Most recently, JMFA [5] and FAN [2] also achieve the state of the art accuracy by leveraging stacked hourglass network. However, these methods do not consider the 'semantic ambiguity' problem which potentially degrades the detection performance.

Two recent works, LAB [26] and SBR [6], are related to this 'semantic ambiguity' problem. By introducing more information than pixel intensity only, they implicitly alle- viate the impact of the annotation noises and improve the performance. LAB [26] trains a facial boundary heatmap estimator and incorporates it into the main landmark regression network. LAB uses the well-defined facial boundaries which provide the facial geometric structure to reduce the ambiguities, leading to improved performance. However, LAB is computational expensive. SBR [6] proposes a registration loss which uses the coherency of optical flow from adjacent frames as its supervision. The additional information from local feature can mitigate the impact of random noises. However, the optical flow is not always credible in unconstrained environment and SBR trains their model on the testing video before the test, limiting its applications. To summarize, LAB and SBR do not intrinsically address the problem of 'semantic ambiguity' because the degraded accuracy is actually derived from the inaccurate labels (human annotations provided by databases). In this work, we solve the 'semantic ambiguity' problem in a more intrinsic way. Specifically, we propose a probabilistic model which can simultaneously search the 'real' ground-truth without semantic ambiguity and train a hourglass landmark detector without using additional information.

\section{Semantic ambiguity}

The semantic ambiguity indicates that some landmarks do not have clear and accurate definition. In this work, we find the semantic ambiguity can happen on any facial points, but mainly on those weak semantic facial points. For example, the landmarks are defined to evenly distribute along the face contour without any clear definition of the exact positions. This ambiguity can potentially affect: (1) the accuracy of the annotations and (2) the convergence of deep model training. For (1), when annotating a database, annotators can introduce random errors to generate inconsistent ground-truths on those weak semantic points due to the lack of clear definitions. For (2), the inconsistent ground-truths generate inconsistent gradients for back-propagation, leading to the difficulty of model convergence. In this section, we qualitatively analyze the influence of semantic ambiguity on landmark detection.

Before this analysis, we briefly introduce our heatmapbased landmark detection network. Specifically, we use a four stage Hourglass (HGs) [17]. It can generate the heatmap which provides the probability of the corresponding landmark located at every pixel, and this probability can facilitate our analysis of semantic ambiguity.

Firstly, we find CNN provides a candidate region rather than a confirmed position for a weak semantic point. In Fig. 2 (a), we can see that the heatmap of a strong semantic point is nearly Gaussian, while the 3D heatmap of a weak semantic point has a 'flat hat', meaning that the confidences in that area are very similar. Since the position with the highest confidence is chosen as the output. The landmark 

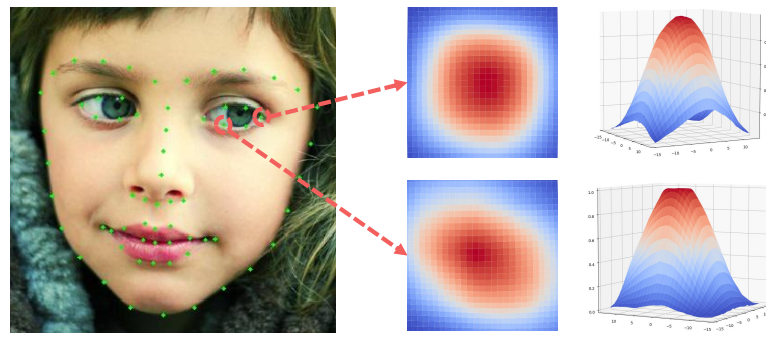

(a) The difference between the heatmap of the eye corner (strong semantic) points and the eye contour (weak semantic) points. Col 2 and 3 represent 2D and 3D heatmaps respectively. In the 3D Gaussian, the $\mathrm{x}, \mathrm{y}$ axes are image coordinates and $\mathrm{z}$ axis is the prediction confidence. We can see the 3D heatmap of a weak semantic point has a 'flat hat'.

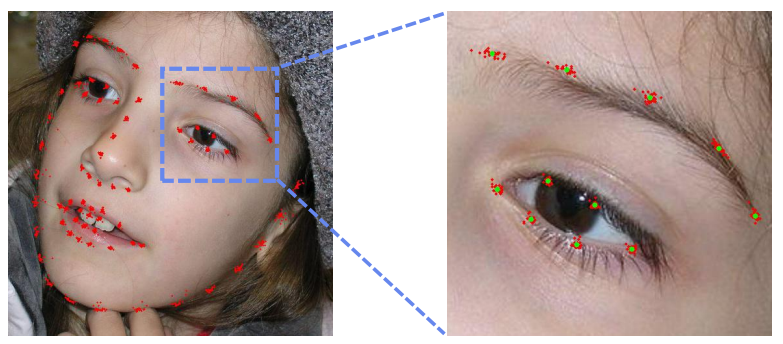

(b) The predictions from a series of checkpoints after convergence When the model has roughly converged, we continue training and achieve the predictions from different iterations. Red and green dots denote the predicted and annotation landmarks, respectively. We can see the predicted landmarks from different checkpoints fluctuate in the neighborhood area of the annotated position (green dots).

Figure 2. The effect of semantic ambiguity

detector tends to output an unexpected random position on the 'flat hat'.

Secondly, we analyze the 'semantic ambiguity' by visualizing how the model is optimized after convergence. When the network has roughly converged, we continue training the network and save a series of checkpoints. In Fig. 2 (b), the eyebrow landmarks, from different checkpoints fluctuate along with the edge of eyebrow, which always generates considerable loss to optimize. However, this loss is ineffectual since the predicted points from different checkpoints also fluctuate in the neighborhood area of the annotated position (green dots in Fig. 2(b)). It can be concluded that the loss caused by random annotation noises dominate the back-propagated gradients after roughly convergence, making the network training trapped into local minima.

\section{Semantically consistent alignment}

In this section, we detail our methodology. In Section 4.1, we model the landmark detection problem using a probabilistic model. To deal with the semantic ambiguity caused by human annotation noise, we introduce a latent variable $\hat{\mathbf{y}}$ which represents the 'real' ground-truth. Then we model the prior model and likelihood in Section 4.2 and 4.3, respectively. Section 4.4 proposes an alternative optimization strategy to search $\hat{\mathbf{y}}$ and train the landmark detector. To recover the unconfidently predicted landmarks due to occlusion and low quality, we propose a global heatmap correction unit (GHCU) in Section 4.5, which refines the predictions by considering the global face shape as a constraint, leading to a more robust model.

\subsection{A probabilistic model of landmark prediction}

In the probabilistic view, training a CNN-based landmark detector can be formulated as a likelihood maximization problem:

$$
\max _{\mathbf{W}} \mathcal{L}(\mathbf{W})=P(\mathbf{o} \mid \mathbf{x} ; \mathbf{W}),
$$

where $\mathbf{o} \in \mathbb{R}^{2 N}$ is the coordinates of the observation of landmarks (e.g. the human annotations). $N$ is the number of landmarks, $\mathbf{x}$ is the input image and $\mathbf{W}$ is the CNN parameters. Under the probabilistic view of Eq. (1), one pixel value on the heatmap works as the confidence of one particular landmark at that pixel. Therefore, the whole heatmap works as the probability distribution over the image.

As analyzed in Section 3, the annotations provided by public databases are usually not fully credible due to the 'semantic ambiguity'. As a result, the annotations, in particular those of weak semantic landmarks, contain random noises and are inconsistent among faces. In this work, we assume that there exists a 'real' ground-truth without semantic ambiguity and can better supervise the network training. To achieve this, we introduce a latent variable $\hat{\mathbf{y}}$ as the 'real' ground-truth which is optimized during learning. Thus, Eq. (1) can be reformulated as:

$$
\begin{aligned}
\max _{\hat{\mathbf{y}}, \mathbf{W}} \mathcal{L}(\hat{\mathbf{y}}, \mathbf{W}) & =P(\mathbf{o}, \hat{\mathbf{y}} \mid \mathbf{x} ; \mathbf{W}) \\
& =P(\mathbf{o} \mid \hat{\mathbf{y}}) P(\hat{\mathbf{y}} \mid \mathbf{x} ; \mathbf{W}),
\end{aligned}
$$

where $\mathbf{o}$ is the observation of $\hat{\mathbf{y}}$, for example, the annotation can be seen as an observation of $\hat{\mathbf{y}}$ from human annotator. $P(\mathbf{o} \mid \hat{\mathbf{y}})$ is a prior of $\hat{\mathbf{y}}$ given the observation $\mathbf{o}$ and $P(\hat{\mathbf{y}} \mid \mathbf{x} ; \mathbf{W})$ is the likelihood.

\subsection{Prior model of 'real' ground-truth}

To optimize Eq. (2), an accurate prior model is important to regularize $\hat{\mathbf{y}}$ and reduce searching space. We assume that the $k$ th landmark $\hat{\mathbf{y}}^{k}$ is close to the $\mathbf{o}^{k}$, which is the observation of $\hat{\mathbf{y}}$. Thus, this prior is modeled as Gaussian similarity over all $\left\{\mathbf{o}^{k}, \hat{\mathbf{y}}^{k}\right\}$ pairs:

$$
\begin{aligned}
P(\mathbf{o} \mid \hat{\mathbf{y}}) & \propto \prod_{k} \exp \left(-\frac{\left\|\mathbf{o}^{k}-\hat{\mathbf{y}}^{k}\right\|^{2}}{2 \sigma_{1}^{2}}\right) \\
& =\exp \left(-\sum_{k} \frac{\left\|\mathbf{o}^{k}-\hat{\mathbf{y}}^{k}\right\|^{2}}{2 \sigma_{1}^{2}}\right),
\end{aligned}
$$




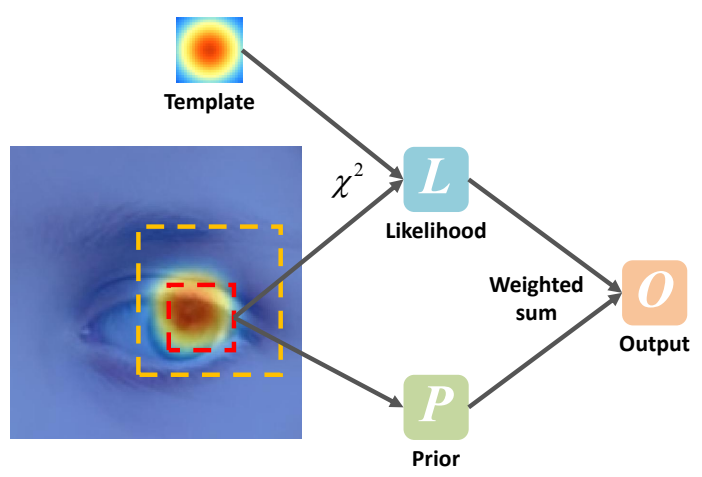

Figure 3. The search of 'real' ground-truth $\hat{\mathbf{y}}$. Yellow and red boxes represent the searching space $\mathcal{N}$ defined in Eq. (7) and the region corresponding to one candidate $\hat{\mathbf{y}}$, respectively. The weighted sum of likelihood and prior is computed as Eq. (8). The search target is to find a position $\hat{\mathbf{y}}$ with the maximum output.

where $\sigma_{1}$ can control the sensitivity to misalignment. To explain $\mathbf{o}^{k}$, we should know in advance that our whole framework is iteratively optimized detailed in Section 4.4. $\mathbf{o}^{k}$ is initialized as the human annotation in the iteration, and will be updated by better observation with iterations.

\subsection{Network likelihood model}

We now discuss the likelihood $P(\hat{\mathbf{y}} \mid \mathbf{x} ; \mathbf{W})$ of Eq. (2). The point-wise joint probability can be represented by the confidence map, which can be modelled by the heatmap of the deep model. Note that our hourglass architecture learns to predict heatmap consisting of a 2D Gaussian centered on the ground-truth $\hat{\mathbf{y}}^{k}$. Thus, for any position $\mathbf{y}$, the more the heatmap region around $\mathbf{y}$ follows a standard Gaussian, the more the pixel at $\mathbf{y}$ is likely to be $\hat{\mathbf{y}}^{k}$. Therefore, the likelihood can be modeled as the distribution distance between the predicted heatmap (predicted distribution) and the standard Gaussian region (expected distribution). In this work, we use Pearson Chi-square test to evaluate the distance of these two distributions:

$$
\chi^{2}(\mathbf{y} \mid \mathbf{x} ; \mathbf{W})=\sum_{i} \frac{\left(\mathbf{E}_{i}-\Phi_{i}(\mathbf{y} \mid \mathbf{x} ; \mathbf{W})\right)^{2}}{\mathbf{E}_{i}}
$$

where $\mathbf{E}$ is a standard Gaussian heatmap (distribution), which is a template representing the ideal response; $i$ is the pixel index; $\Phi$ is a cropped patch (of the same size as Gaussian template) from the predicted heatmap centered on $\mathbf{y}$. Finally, the joint probability can also be modeled as a product of Gaussian similarities maximized over all landmarks:

$$
P(\hat{\mathbf{y}} \mid \mathbf{x} ; \mathbf{W})=\exp \left(-\sum_{k} \frac{\chi_{k}^{2}(\hat{\mathbf{y}} \mid \mathbf{x} ; \mathbf{W})}{2 \sigma_{2}^{2}}\right)
$$

where $k$ is the landmark index, $\sigma_{2}$ is the bandwidth of likelihood.
To keep the likelihood credible, we first train a network with the human annotations. Then in the likelihood, we can consider the trained network as a super annotator to guide the searching of the real ground-truth. It results from the fact that a well trained network is able to capture the statistical law of annotation noise from the whole training set, so that it can generate predictions with better semantic consistency.

\subsection{Optimization}

Combining Eq. (2), (3) and (5) and taking log of the likelihood, we have:

$$
\log \mathcal{L}(\hat{\mathbf{y}}, \mathbf{W})=\sum_{k}\left(-\frac{\left\|\mathbf{o}^{k}-\hat{\mathbf{y}}^{k}\right\|^{2}}{2 \sigma_{1}^{2}}-\frac{\chi^{2}(\hat{\mathbf{y}} \mid \mathbf{x} ; \mathbf{W})}{2 \sigma_{2}^{2}}\right)
$$

Reduce Searching Space To optimize the latent semantically consistent 'real' landmark $\hat{\mathbf{y}}^{k}$, the prior Eq. 33 indicates that the latent 'real' landmark is close to the observed landmark $\mathbf{o}^{k}$. Therefore, we reduce the search space of $\hat{\mathbf{y}}^{k}$ to a small patch centered on $\mathbf{o}^{k}$. Then, the optimization problem of Eq. (6) can be re-written as:

$$
\begin{array}{r}
\min _{\hat{\mathbf{y}}, \mathbf{W}}-\log \mathcal{L}(\hat{\mathbf{y}}, \mathbf{W}) \\
\text { s.t. } \hat{\mathbf{y}}^{k} \in \mathcal{N}\left(\mathbf{o}^{k}\right)
\end{array}
$$

where $\mathcal{N}\left(\mathbf{o}^{k}\right)$ represents a region centered on $\mathbf{o}^{k}$.

Alternative Optimization To optimize Eq. (7), an alternative optimization strategy is applied. In each iteration, $\hat{\mathbf{y}}$ is firstly searched with the network parameter $\mathbf{W}$ fixed. Then $\hat{\mathbf{y}}$ is fixed and $\mathbf{W}$ is updated (landmark prediction network training) under the supervision of newly searched $\hat{\mathbf{y}}$.

Step 1: When $\mathbf{W}$ is fixed, to search the latent variable $\hat{\mathbf{y}}$, the optimization becomes a constrained discrete optimization problem for each landmark:

$$
\min _{\hat{\mathbf{y}}^{k}}\left(\frac{\left\|\mathbf{o}^{k}-\hat{\mathbf{y}}^{k}\right\|^{2}}{2 \sigma_{1}^{2}}+\frac{\chi^{2}\left(\hat{\mathbf{y}}^{k} \mid \mathbf{x} ; \mathbf{W}\right)}{2 \sigma_{2}^{2}}\right)
$$

where all the variables are known except $\hat{\mathbf{y}}^{k}$. We search $\hat{\mathbf{y}}^{k}$ by going through all the pixels in $\mathcal{N}\left(\mathbf{o}^{k}\right)$ (a neighborhood area of $\mathbf{o}^{k}$ as shown in Fig. 3 and the one with minimal loss in Eq. (8) is the solution. Since the searching space $\mathcal{N}\left(\mathbf{o}^{k}\right)$ is very small, i.e. $17 \times 17$ in this work for $256 \times 256$ heatmap, the optimization is very efficient.

Note that in the prior part of Eq. (8), $\mathbf{o}^{k}$ is the observation of $\hat{\mathbf{y}}^{k}$ : In the 1 st iteration, $\mathbf{o}^{k}$ is set to the human annotations which are the observation of human annotators; From the 2nd iteration, $\mathbf{o}^{k}$ is set to $\hat{\mathbf{y}}_{t-1}^{k}$ (where $t$ is the iteration). Note that $\hat{\mathbf{y}}_{t-1}^{k}$ is the estimated 'real' ground-truth 

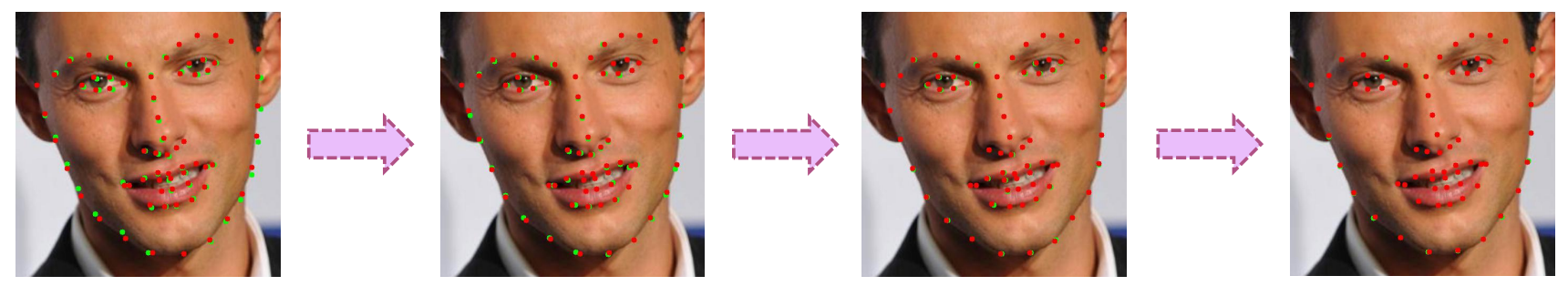

Figure 4. Gradual convergence (one image represents one iteration) from the observation o (i.e. $\hat{\mathbf{y}}$ of the last iteration, green dots) to the estimate of real ground-truth $\hat{\mathbf{y}}$ (red dots). For last image, the optimization converges because red and green dots are completely overlapped.

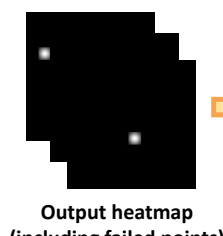

(including failed points)

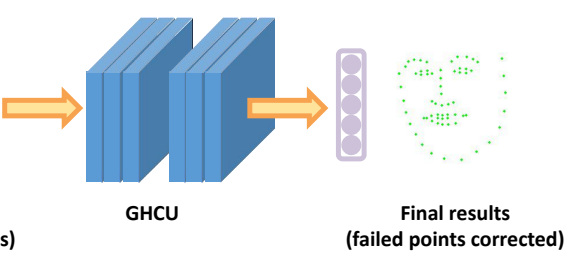

(a) The use of GHCU for correcting some failed points.
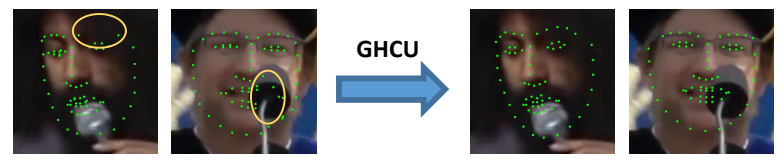

(b) Correcting challenging points with GHCU on 300-VW.

Figure 5. Global Heatmap Correction Unit (GHCU)

from the last iteration. With the iterations, $\hat{\mathbf{y}}_{t}^{k}$ is converging to the 'real' ground-truth because both the current observation $\mathbf{o}^{k}$ (i.e. $\hat{\mathbf{y}}_{t-1}^{k}$ ) and $\mathrm{CNN}$ prediction iteratively become more credible.

Step 2: When $\hat{\mathbf{y}}$ is fixed, the optimization becomes:

$$
\min _{\mathbf{W}} \sum_{k} \frac{\chi^{2}\left(\hat{\mathbf{y}}^{k} \mid \mathbf{x} ; \mathbf{W}\right)}{2 \sigma_{2}^{2}}
$$

The optimization becomes a typical network training process under the supervision of $\hat{\mathbf{y}}$. Here $\hat{\mathbf{y}}$ is set to the estimate of the latent 'real' ground-truth obtained in Step 1. Figure 4 shows an example of the gradual convergence from the observation $\mathbf{o}(\hat{\mathbf{y}}$ of the last iteration) to the estimate of real ground-truth $\hat{\mathbf{y}}$. The optimization of $\hat{\mathbf{y}}$ in our semantic alignment can easily converge to a stable position, which does not have hard convergence problem like the traditional landmark training as shown in Fig. 2 p.

\subsection{Global heatmap correction unit}

Traditional heatmap based methods predict each landmark as an individual task without considering global face shape. The prediction might fail when the model fits images of low-quality and occlusion as shown in Fig. $5 \mathrm{~b}$. The outliers such as occlusions destroy the face shape and significantly reduce overall performance.
Table 1. GHCU Architecture ( $N$ is the number of the landmarks)

\begin{tabular}{c|c|c}
\hline Layers & Output size & GHCU \\
\hline Conv1 & $128 \times 128$ & {$[5 \times 5,64]$, stride 2} \\
Conv2 & $64 \times 64$ & {$[3 \times 3,64]$, stride 2} \\
Conv3 & $32 \times 32$ & {$[3 \times 3,32]$, stride 2} \\
Conv4 & $16 \times 16$ & {$[3 \times 3,32]$, stride 2} \\
Conv5 & $8 \times 8$ & {$[3 \times 3,16]$, stride 2 } \\
Conv6 & $4 \times 4$ & {$[3 \times 3,16]$, stride 2 } \\
\hline FC1 & - & 256 \\
FC2 & - & $2 N$ \\
\hline
\end{tabular}

Existing methods like local feature based CLM [4] and deep learning based LGCN [14] apply a 2D shape PCA as their post-processing step to remove the outliers. However, PCA based method is weak to model out-of-plane rotation and very slow (about $0.8 \mathrm{fps}$ in LGCN [14]). In this work, we propose a Global Heatmap Correction Unit (GHCU) to recover the outliers efficiently. We view the predicted heatmaps as input and directly regress the searched/optimized $\hat{\mathbf{y}}$ through a light weight $\mathrm{CNN}$ as shown in Tab. 1. The GHCU implicitly learns the whole face shape constraint from the training data and always gives facialshape landmarks, as shown in Fig. 5 Our experiments demonstrate the GHCU completes fitting with the speed 8 times faster than PCA on the same hardware platform and achieves higher accuracy than PCA.

\section{Experiments}

Datesets. We conduct evaluation on three challenging datasets including image based 300W [21], AFLW [9], and video based 300-VW [22, 24, 3].

$300 \mathrm{~W}[21]$ is a collection of multiple face datasets, including the LFPW [1], HELEN [11], AFW [19] and XM2VTS [15] which have 68 landmarks. The training set contains 3148 training samples, 689 testing samples which are further divided into the common and challenging subsets.

AFLW [9] is a very challenging dataset which has a wide range of pose variations in yaw $\left(-90^{\circ}\right.$ to $\left.90^{\circ}\right)$. In this work, we follow the AFLW-Full protocol [33] which ignores two landmarks of ears and use the remaining 19 landmarks.

$300-V W$ [22, 24, 3] is a large dataset for video-based face alignment, which consists of 114 videos in various 
conditions. Following [22], we utilized all images from $300 \mathrm{~W}$ and 50 sequences for training and the remaining 64 sequences for testing. The test set consists of three categories: well-lit, mild unconstrained and challenging.

Evaluation metric. To compare with existing popular methods, we conduct different evaluation metrics on different datasets. For $300 \mathrm{~W}$ dataset, We follow the protocol in [20] and use Normalized mean errors (NME) which normalizes the error by the inter-pupil distance. For AFLW, we follow [32] to use face size as the normalizing factor. For 300-VW dataset, we employed the standard normalized root mean squared error (RMSE) [22] which normalizes the error by the outer eye corner distance.

Implementation Details. In our experiments, all the training and testing images are cropped and resized to $256 \times 256$ according to the provided bounding boxes. To perform data augmentation, we randomly sample the angle of rotation and the bounding box scale from Gaussian distribution. We use a four-stage stacked hourglass network [17] as our backbone which is trained by the optimizer RMSprop. As described in Section 4, our algorithm comprises two parts: network training and real groundtruth searching, which are alternatively optimized. Specifically, at each epoch, we first search the real ground-truth $\hat{\mathbf{y}}$ and then use $\hat{\mathbf{y}}$ to supervise the network training. When training the roughly converged model with human annotations, the initial learning rate is $2.5 \times 10^{-4}$ which is decayed to $2.5 \times 10^{-6}$ after 120 epochs. When training with Semantic Alignment from the beginning of the aforementioned roughly converged model, the initial learning rate is $2.5 \times 10^{-6}$ and is divided by 5,2 and 2 at epoch 30,60 and 90 respectively. During semantic alignment, we search the latent variable $\hat{\mathbf{y}}$ from a $17 \times 17$ region centered at the current observation point $\mathbf{o}$, and we crop a no larger than $25 \times 25$ patch from the predicted heatmap around current position for Pearson Chi-square test in Eq. (4). We set batch size to 10 for network training. For GHCU, the network architecture is shown in Tab. 1 All our models are trained with PyTorch [18] on 2 Titan X GPUs.

\subsection{Comparison experiment}

300W. We compare our approach against the state-ofthe-art methods on 300W in Tab. 2. The baseline (HGs in Tab. 2) uses the hourglass architecture with human annotations, which is actually the traditional landmark detector training. From Tab.2 2 we can see that HGs with our Semantic Alignment (HGs + SA) greatly outperform hourglass (HGs) only, $4.37 \%$ vs $5.04 \%$ in terms of NME on Full set, showing the great effectiveness of our Semantic Alignment (SA). By adding GHCU, we can see that $\mathrm{HGs}+\mathrm{SA}+\mathrm{GHCU}$ slightly outperforms the HGs+SA. The improvement is not significant because the images of $300 \mathrm{~W}$ are of high resolution, while GHCU works particularly well for images of low resolution and occlusions verified in the following
Table 2. Comparisons with state of the art on $300 \mathrm{~W}$ dataset. The error (NME) is normalized by the inter-pupil distance.

\begin{tabular}{l|ccc}
\hline Method subset & Com. & Challenge & Full \\
\hline SDM [28] & 5.60 & 15.40 & 7.52 \\
CFSS [32] & 4.73 & 9.98 & 5.76 \\
TCDCN [30] & 4.80 & 8.60 & 5.54 \\
LBF [20] & 4.95 & 11.98 & 6.32 \\
3DDFA (CVPR16) [35] & 6.15 & 10.59 & 7.01 \\
3DDFA + SDM & 5.53 & 9.56 & 6.31 \\
RAR (ECCV16) [27] & 4.12 & 8.35 & 4.94 \\
TR-DRN (CVPR17) [13] & 4.36 & 7.56 & 4.99 \\
Wing (CVPR18) [7] & $\mathbf{3 . 2 7}$ & 7.18 & 4.04 \\
LAB (CVPR18) [26] & 3.42 & 6.98 & 4.12 \\
SBR (CVPR18) [6] & 3.28 & 7.58 & 4.10 \\
PCD-CNN (CVPR18) [10] & 3.67 & 7.62 & 4.44 \\
DCFE (ECCV18) [25] & 3.83 & 7.54 & 4.55 \\
\hline HGs & 4.43 & 7.56 & 5.04 \\
HGs + SA & 3.75 & 6.90 & 4.37 \\
HGs + SA + GHCU & 3.74 & 6.87 & 4.35 \\
HGs + Norm & 3.95 & 6.51 & 4.45 \\
HGs + SA + Norm & 3.46 & 6.38 & 4.03 \\
HGs + SA + Norm + GHCU & 3.45 & $\mathbf{6 . 3 8}$ & $\mathbf{4 . 0 2}$ \\
\hline
\end{tabular}

evaluations. Following [7] and [29] which normalize the in-plane-rotation by training a preprocessing network, we conduct this normalization (HGs+SA+GHCU+Norm) and achieve state of the art performance on Challenge set and Full set: $6.38 \%$ and $4.02 \%$. In particular, on Challenge set, we significantly outperform the state of the art method: $6.38 \%$ (HGs+SA+GHCU+Norm) vs $6.98 \%$ (LAB), meaning that our method is particularly effective on challenging scenarios.

AFLW. 300W has 68 facial points which contain many weak semantic landmarks (e.g. those on face contours). In comparison, AFLW has only 19 points, most of which are strong semantic landmarks. Since our SA is particularly effective on weak semantic points, we conduct experiments on AFLW to verify whether SA generalizes well to the point set, most of which are strong semantic points. For fair comparison, we do not compare methods using additional outside training data, e.g. LAB [26] used additional boundary information from outside database. As shown in Tab. 3 . HGs+SA outperforms HGs, $1.62 \%$ vs $1.95 \%$. It means that even though corner points are easily to be recognized, there is still random error in annotation, which can be corrected by SA. It is also observed that $\mathrm{HGs}+\mathrm{SA}+\mathrm{GHCU}$ works better than HGs+SA.

300-VW. Unlike the image-based databases $300 \mathrm{~W}$ and AFLW, 300-VW is video-based database, which is more challenging because the frame is of low resolution and with strong occlusions. The subset Category 3 is the most challenging one. From Tab. 4, we can see that HGs + SA greatly outperforms HGs in each of these three test sets. Furthermore, compared with HGs + SA, HGs + SA + GHCU reduce the error rate (RMSE) by $18 \%$ on Category 3 test set, meaning that GHCU is very effective for video-based challenges such as low resolution and occlusions because 
Table 3. Comparison with state of the art on AFLW dataset. The error (NME) is normalized by the face bounding box size.

\begin{tabular}{l|c}
\hline Method & AFLW-Full (\%) \\
\hline LBF [20] & 4.25 \\
CFSS [32] & 3.92 \\
CCL (CVPR16) [33] & 2.72 \\
TSR (CVPR17) [13] & 2.17 \\
DCFE (ECCV18) [25] & 2.17 \\
SBR (CVPR18) [6] & 2.14 \\
DSRN (CVPR18) [16] & 1.86 \\
Wing (CVPR18) [7] & 1.65 \\
\hline HGs & 1.95 \\
HGs + SA & 1.62 \\
HGs + SA + GHCU & $\mathbf{1 . 6 0}$ \\
\hline
\end{tabular}

GHCU considers the global face shape as constraint, being robust to such challenging factors.

Table 4. Comparison with state of the art on 300-VW dataset. The error (RMSE) is normalized by the inter-ocular distance.

\begin{tabular}{l|ccc}
\hline Method & Category 1 & Category 2 & Category 3 \\
\hline SDM [28] & 7.41 & 6.18 & \\
CFSS [32] & 7.68 & 6.42 & 13.67 \\
TCDCN [31] & 7.66 & 6.77 & 14.98 \\
TSTN [12] & 5.36 & 4.51 & 12.84 \\
DSRN (CVPR18) [16] & 5.33 & 4.92 & 8.85 \\
\hline HGs & 4.32 & 3.83 & 9.91 \\
HGs + SA & 4.06 & 3.58 & 9.19 \\
HGs + SA + GHCU & $\mathbf{3 . 8 5}$ & $\mathbf{3 . 4 6}$ & $\mathbf{7 . 5 1}$ \\
\hline
\end{tabular}

\subsection{Self evaluations}

Balance of prior and likelihood As shown in Eq. (6), the 'real' ground-truth is optimized using two parts: prior and likelihood, where $\sigma_{1}$ and $\sigma_{2}$ determine the importance of these two parts. Thus, we can use one parameter $\sigma_{2}^{2} / \sigma_{1}^{2}$ to estimate this importance weighting. We evaluate different values of $\sigma_{2}^{2} / \sigma_{1}^{2}$ in Tab. 5. Clearly, the performance of $\sigma_{2}^{2} / \sigma_{1}^{2}=0$ (removing Semantic Alignment and using human annotations only) is worst, showing the importance of the proposed Semantic Alignment. We find that $\sigma_{2}^{2} / \sigma_{1}^{2}=0.1$ achieves the best performance, meaning that the model relies much more (10 times) on prior than likelihood to achieve the best trade-off.

Table 5. The effect of the ratio $\sigma_{2}^{2} / \sigma_{1}^{2}$ in Eq. 8 \% on 300W.

\begin{tabular}{l|ccccccc}
\hline$\sigma_{2}^{2} / \sigma_{1}^{2}$ & 0 & 0.01 & 0.05 & 0.1 & 0.3 & 0.5 & 1 \\
\hline NME (\%) & 4.99 & 4.79 & 4.40 & $\mathbf{4 . 3 7}$ & 4.46 & 4.54 & 4.68 \\
\hline
\end{tabular}

Template size. As discussed in the Section 3, for a position $\mathbf{y}$, the similarity between the heatmap region around it and standard Gaussian template is closely related to the detection confidence. Therefore, the size of the Gaussian template, which is used to measure the network confidence in Eq. (5), can affect the final results. Table 6 reports the results under different template sizes using the model HGs+SA. Too small size (size $=1$ ) means that the heatmap value is directly used to model the likelihood instead of Chi- square test. Not surprisingly, the performance with size $=1$ is not promising. Large size ( $\operatorname{size}=25$ ) introduces more useless information, degrading the performance. In our experiment, we find size $=15$ for AFLW and size $=19$ for $300 \mathrm{~W}$ can achieve the best result.

Table 6. The effects of template size on 300W and AFLW test sets.

\begin{tabular}{l|cccccc}
\hline template size & 1 & 7 & 11 & 15 & 19 & 25 \\
\hline 300W Full(\%) & 4.76 & 4.72 & 4.61 & 4.53 & $\mathbf{4 . 3 7}$ & 4.43 \\
\hline AFLW Full (\%) & 1.89 & 1.80 & 1.72 & $\mathbf{1 . 6 2}$ & 1.66 & 1.70 \\
\hline
\end{tabular}

Analysis of the training of semantic alignment. To verify the effectiveness of Semantic Alignment, we train a baseline network using hourglass under the supervision of human annotation to converge. Use this roughly converged baseline, we continue training using 3 strategies as shown in Fig. 6 and 7. baseline, SA w/o update (always using human annotation as the observation, see Eq. (6) and SA (the observation is iteratively updated). Fig. 6 and 77 visualize the changes of training loss and NME on test set against the training epochs, respectively. We can see that the baseline curve in Fig. 6 and 7 do not decrease because of the 'semantic ambiguity'. By introducing SA, the training loss and test NME steadily drop. Obviously, SA reduces the random optimizing directions and helps the roughly converged network to further improve the detection accuracy.

We also evaluate the condition that uses semantic alignment without updating the observation o ('SA w/o update' in Fig. 6 and 77. It means o is always set to the human annotations. We can see that the curve of 'SA w/o update' can be further optimized but quickly trapped into local optima, leading to worse performance than SA. We assume that the immutable observation o reduces the capacity of searching 'real' ground-truth $\hat{\mathbf{y}}$.

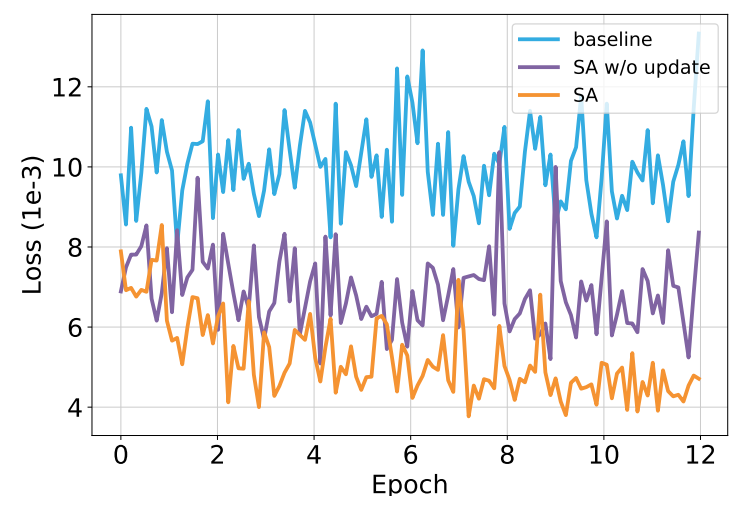

Figure 6. Training loss of the baseline, Semantic Alignment without updating observation (SA w/o update) and Semantic Alignment (SA). The training starts at a roughly converged model (trained using human annotations only) using $300 \mathrm{~W}$ training set.

The update of Semantic Alignment. Under Semantic Alignment framework, all the training labels are updated 


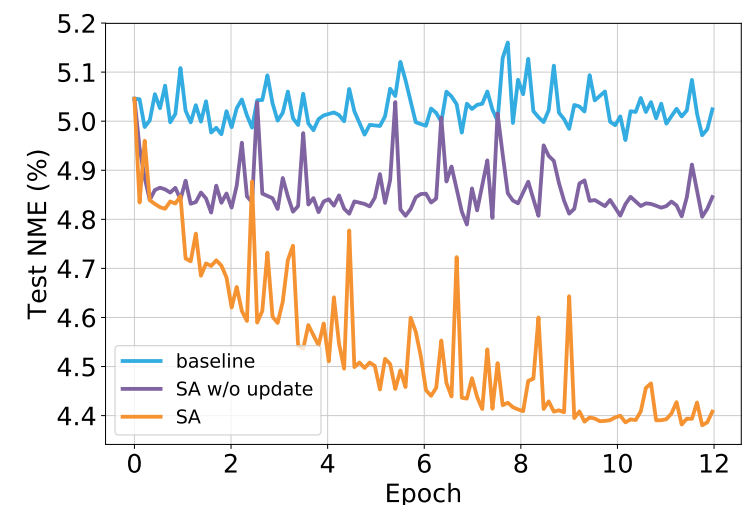

Figure 7. NME of the baseline, Semantic Alignment without updating observation (SA w/o update) and Semantic Alignment (SA). The training starts at a roughly converged model (trained using human annotations only) on $300 \mathrm{~W}$ full test set.

after each epoch. To explore the effects of the number of epochs on model convergence, we train different models by stopping semantic alignment at different epochs. In Fig 8 , it is observed that the final performance keeps improving with the times of semantic alignment, which demonstrates that the improvement is highly positive related to the quality of the learned $\hat{\mathbf{y}}$. From our experiment, 10 epochs of semantic alignment are enough for our data sets.

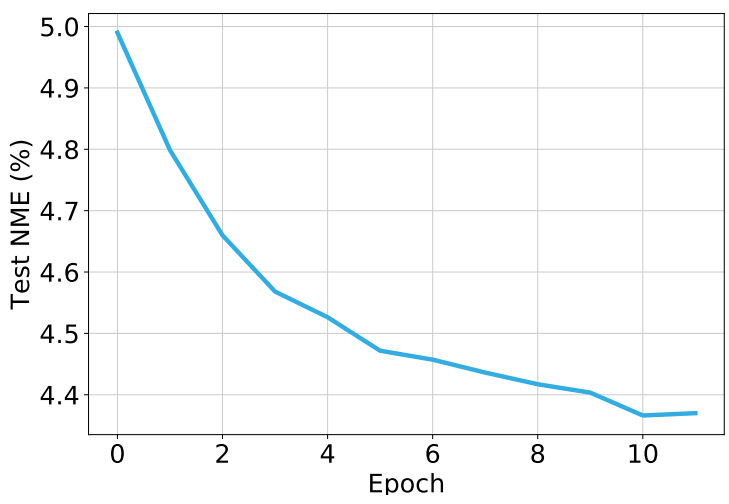

Figure 8. NME vs Semantic Alignment update epochs on 300W full test set

Quality of the searched 'real' ground-truth. One important assumption of this work is that there exist 'real' ground-truths which are better than the human annotations. To verify this, we train two networks which are supervised by the human annotations provided by public database and the searched 'real' ground-truth, respectively. These two detectors are a Hourglass model (HGs) and a ResNet [8] model which directly regresses the landmark coordinates as [7]. As shown in Tab. 7, we can see that on both models the 'real' ground-truth (SA) outperforms the human annotations (HA). Clearly, our learned labels are better than the human annotations, verifying our assumption that the se- mantic alignment can find the semantic consistent groundtruths.

Table 7. The comparison of the labels searched by our Semantic Alignment (SA) and human annotations (HA) on 300w-full set

\begin{tabular}{l|cccc}
\hline Method & HGs (HA) & HGs (SA) & Reg (HA) & Reg (SA) \\
\hline NME (\%) & 5.04 & $\mathbf{4 . 3 7}$ & 5.49 & $\mathbf{5 . 1 2}$ \\
\hline
\end{tabular}

Global heatmap correction unit. The 2D shape PCA can well keep the face constraint and can be conducted as a post-processing step to enhance the performance of heatmap based methods, like CLM [4] and most recently LGCN [14]. We apply the powerful PCA refinement method in LGCN and compare it with our GHCU. We evaluate on 300-VW where the occlusion and low-quality are particularly challenging. As shown in Tab. 8, our CNN based GHCU outperforms PCA based method in terms of both accuracy and efficiency.

Table 8. The comparison of GHCU with traditional PCA-based refinement on $300-\mathrm{VW}$ database.

\begin{tabular}{l|cccc}
\hline Method & Category 1 & Category 2 & Category 3 & CPU Time (ms) \\
\hline Baseline & 4.06 & 3.58 & 9.19 & - \\
PCA [14] & 3.99 & $\mathbf{3 . 2 6}$ & 7.69 & 1219 \\
GHCU & $\mathbf{3 . 8 5}$ & 3.46 & $\mathbf{7 . 5 1}$ & 149 \\
\hline
\end{tabular}

Ablation study. To verify the effectiveness of different components in our framework, we conduct this ablation study on $300-\mathrm{VW}$. For a fair comparison, all the experiments use the same parameter settings. As shown in Tab.9. Semantic alignment can consistently improve the performance on all subset sets, demonstrating the strong generalization capacity of SA. GHCU is more effective on the challenge data set (Category 3): $8.15 \%$ vs $9.91 \%$; Combining SA and GHCU works better than single of them, showing the complementary of these two mechanisms.

Table 9. Effectiveness of SA and GHCU tested on 300-VW.

\begin{tabular}{l|cccc}
\hline Semantic Alignment (SA) & $\checkmark$ & & $\checkmark$ & \\
GHCU & $\checkmark$ & $\checkmark$ & & \\
\hline Category 1 & $\mathbf{3 . 8 5}$ & 4.03 & 4.06 & 4.32 \\
Category 2 & $\mathbf{3 . 4 6}$ & 3.66 & 3.58 & 3.83 \\
Category 3 & $\mathbf{7 . 5 1}$ & 8.15 & 9.19 & 9.91 \\
\hline
\end{tabular}

\section{Conclusion}

In this paper, we first analyze the semantic ambiguity of facial landmarks and show that the potential random noises of landmark annotations can degrade the performance considerably. To address this issue, we propose a a novel latent variable optimization strategy to find the semantically consistent annotations and alleviate random noises during training stage. Extensive experiments demonstrated that our method effectively improves the landmark detection accuracy on different data sets. 


\section{References}

[1] P. N. Belhumeur, D. W. Jacobs, D. J. Kriegman, and N. Kumar. Localizing parts of faces using a consensus of exemplars. In Computer Vision and Pattern Recognition, pages 545-552, 2011.

[2] Adrian Bulat and Georgios Tzimiropoulos. How far are we from solving the $2 \mathrm{~d} \& 3 \mathrm{~d}$ face alignment problem?(and a dataset of 230,000 3d facial landmarks). In International Conference on Computer Vision, volume 1, page 4, 2017.

[3] Grigoris G Chrysos, Epameinondas Antonakos, Stefanos Zafeiriou, and Patrick Snape. Offline deformable face tracking in arbitrary videos. In Proceedings of the IEEE International Conference on Computer Vision Workshops, pages 1-9, 2015.

[4] David Cristinacce and Tim Cootes. Automatic feature localisation with constrained local models. Pattern Recognition, 41(10):3054-3067, 2008.

[5] Jiankang Deng, George Trigeorgis, Yuxiang Zhou, and Stefanos Zafeiriou. Joint multi-view face alignment in the wild. 2017.

[6] Xuanyi Dong, Shoou-I Yu, Xinshuo Weng, Shih-En Wei, Yi Yang, and Yaser Sheikh. Supervision-by-registration: An unsupervised approach to improve the precision of facial landmark detectors. In Proceedings of the IEEE Conference on Computer Vision and Pattern Recognition, pages 360-368, 2018.

[7] Zhen-Hua Feng, Josef Kittler, Muhammad Awais, Patrik Huber, and Xiao-Jun Wu. Wing loss for robust facial landmark localisation with convolutional neural networks. arXiv preprint arXiv:1711.06753, 2017.

[8] Kaiming He, Xiangyu Zhang, Shaoqing Ren, and Jian Sun. Deep residual learning for image recognition. In Proceedings of the IEEE conference on computer vision and pattern recognition, pages 770-778, 2016.

[9] Martin Koestinger, Paul Wohlhart, Peter M Roth, and Horst Bischof. Annotated facial landmarks in the wild: A largescale, real-world database for facial landmark localization. In Computer Vision Workshops (ICCV Workshops), 2011 IEEE International Conference on, pages 2144-2151. IEEE, 2011.

[10] Amit Kumar and Rama Chellappa. Disentangling 3d pose in a dendritic cnn for unconstrained $2 \mathrm{~d}$ face alignment. In Proceedings of the IEEE Conference on Computer Vision and Pattern Recognition, pages 430-439, 2018.

[11] Vuong Le, Jonathan Brandt, Zhe Lin, Lubomir Bourdev, and Thomas S. Huang. Interactive facial feature localization. In European Conference on Computer Vision, pages 679-692, 2012.

[12] Hao Liu, Jiwen Lu, Jianjiang Feng, and Jie Zhou. Twostream transformer networks for video-based face alignment. IEEE Transactions on Pattern Analysis \& Machine Intelligence, (1):1-1, 2017.

[13] Jiang-Jing Lv, Xiaohu Shao, Junliang Xing, Cheng Cheng, Xi Zhou, et al. A deep regression architecture with twostage re-initialization for high performance facial landmark detection. In $C V P R$, volume 1, page 4, 2017.
[14] Daniel Merget, Matthias Rock, and Gerhard Rigoll. Robust facial landmark detection via a fully-convolutional localglobal context network. In Proceedings of the IEEE Conference on Computer Vision and Pattern Recognition, pages 781-790, 2018.

[15] K. Messer, J. Matas, J. Kittler, and K. Jonsson. Xm2vts: the extended m2vts database. In Proc. Second International Conference on Audio- and Video-Based Biometric Person Authentication, pages 72-77, 2000.

[16] Xin Miao, Xiantong Zhen, Xianglong Liu, Cheng Deng, Vassilis Athitsos, and Heng Huang. Direct shape regression networks for end-to-end face alignment. In Proceedings of the IEEE Conference on Computer Vision and Pattern Recognition, pages 5040-5049, 2018.

[17] Alejandro Newell, Kaiyu Yang, and Jia Deng. Stacked hourglass networks for human pose estimation. In European Conference on Computer Vision, pages 483-499. Springer, 2016.

[18] Adam Paszke, Sam Gross, Soumith Chintala, Gregory Chanan, Edward Yang, Zachary DeVito, Zeming Lin, Alban Desmaison, Luca Antiga, and Adam Lerer. Automatic differentiation in pytorch. 2017.

[19] Deva Ramanan. Face detection, pose estimation, and landmark localization in the wild. In IEEE Conference on Computer Vision and Pattern Recognition, pages 2879-2886, 2012.

[20] Shaoqing Ren, Xudong Cao, Yichen Wei, and Jian Sun. Face alignment via regressing local binary features. IEEE Transactions on Image Processing, 25(3):1233-1245, 2016.

[21] Christos Sagonas, Georgios Tzimiropoulos, Stefanos Zafeiriou, and Maja Pantic. A semi-automatic methodology for facial landmark annotation. In Computer Vision and Pattern Recognition Workshops, pages 896-903, 2013.

[22] Jie Shen, Stefanos Zafeiriou, Grigoris G Chrysos, Jean Kossaifi, Georgios Tzimiropoulos, and Maja Pantic. The first facial landmark tracking in-the-wild challenge: Benchmark and results. In Proceedings of the IEEE International Conference on Computer Vision Workshops, pages 50-58, 2015.

[23] Yi Sun, Xiaogang Wang, and Xiaoou Tang. Deep convolutional network cascade for facial point detection. In Computer Vision and Pattern Recognition, pages 3476-3483, 2013.

[24] Georgios Tzimiropoulos. Project-out cascaded regression with an application to face alignment. In Proceedings of the IEEE Conference on Computer Vision and Pattern Recognition, pages 3659-3667, 2015.

[25] Roberto Valle and M José. A deeply-initialized coarse-tofine ensemble of regression trees for face alignment. In Proceedings of the European Conference on Computer Vision (ECCV), pages 585-601, 2018.

[26] Wayne Wu, Chen Qian, Shuo Yang, Quan Wang, Yici Cai, and Qiang Zhou. Look at boundary: A boundary-aware face alignment algorithm. In Proceedings of the IEEE Conference on Computer Vision and Pattern Recognition, pages 21292138, 2018.

[27] Shengtao Xiao, Jiashi Feng, Junliang Xing, Hanjiang Lai, Shuicheng Yan, and Ashraf Kassim. Robust facial landmark detection via recurrent attentive-refinement networks. 
In European conference on computer vision, pages 57-72. Springer, 2016.

[28] Xuehan Xiong and Fernando De La Torre. Supervised descent method and its applications to face alignment. In Computer Vision and Pattern Recognition, pages 532-539, 2013.

[29] Jing Yang, Qingshan Liu, and Kaihua Zhang. Stacked hourglass network for robust facial landmark localisation. In IEEE Conference on Computer Vision and Pattern Recognition Workshops, pages 2025-2033, 2017.

[30] Zhanpeng Zhang, Ping Luo, Change Loy Chen, and Xiaoou Tang. Facial landmark detection by deep multi-task learning. In European Conference on Computer Vision, pages 94-108, 2014.

[31] Zhanpeng Zhang, Ping Luo, Chen Change Loy, and Xiaoou Tang. Learning deep representation for face alignment with auxiliary attributes. IEEE transactions on pattern analysis and machine intelligence, 38(5):918-930, 2016.

[32] Shizhan Zhu, Cheng Li, Chen Change Loy, and Xiaoou Tang. Face alignment by coarse-to-fine shape searching. In Proceedings of the IEEE Conference on Computer Vision and Pattern Recognition, pages 4998-5006, 2015.

[33] Shizhan Zhu, Cheng Li, Chen-Change Loy, and Xiaoou Tang. Unconstrained face alignment via cascaded compositional learning. In Proceedings of the IEEE Conference on Computer Vision and Pattern Recognition, pages 34093417, 2016.

[34] Xiangyu Zhu, Zhen Lei, Stan Z Li, et al. Face alignment in full pose range: A 3d total solution. IEEE Transactions on Pattern Analysis and Machine Intelligence, 2017.

[35] Xiangyu Zhu, Zhen Lei, Xiaoming Liu, Hailin Shi, and Stan Z. Li. Face alignment across large poses: A 3d solution. In IEEE Conference on Computer Vision and Pattern Recognition, pages 146-155, 2016. 